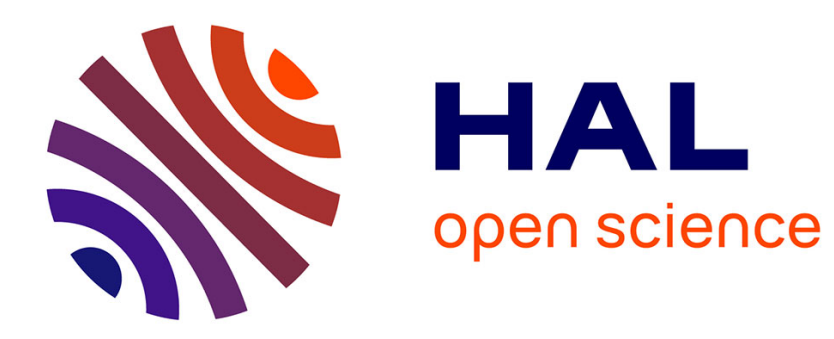

\title{
On-the-field calibration of an array of sensors
}

Eric Dorveaux, David Vissière, Nicolas Petit

\section{To cite this version:}

Eric Dorveaux, David Vissière, Nicolas Petit. On-the-field calibration of an array of sensors. 2010 American Control Conference, Jun 2010, Baltimore, United States. pp.6795-6802. hal-00525783

\section{HAL Id: hal-00525783 \\ https: / hal-mines-paristech.archives-ouvertes.fr/hal-00525783}

Submitted on 12 Oct 2010

HAL is a multi-disciplinary open access archive for the deposit and dissemination of scientific research documents, whether they are published or not. The documents may come from teaching and research institutions in France or abroad, or from public or private research centers.
L'archive ouverte pluridisciplinaire HAL, est destinée au dépôt et à la diffusion de documents scientifiques de niveau recherche, publiés ou non, émanant des établissements d'enseignement et de recherche français ou étrangers, des laboratoires publics ou privés. 


\title{
On-the-field calibration of an array of sensors
}

\author{
Eric Dorveaux, David Vissière, Nicolas Petit
}

\begin{abstract}
We address the problem of the calibration of an array of sensors by investigating theoretically and experimentally the case of 2 three-axis sensors. Our focus is on magnetometers that can be used in a low-cost inertial navigation system. Usual errors (misalignments, non-orthogonality, scale factors, biases) are accounted for. The proposed calibration method requires no specific calibration hardware. Instead, we solely use the fact that, if the sensor is properly calibrated, the norm of the sensed field must remain constant irrespective of the sensors orientation. Several strategies of calibration for an array of sensors are described along with the impact of (unavoidable) field disturbances. Experiments conducted with a couple of magneto-resistive magnetometers and data fusion results illustrate the relevance of the approach.
\end{abstract}

\section{INTRODUCTION}

Recently an innovative approach of inertial navigation has been proposed to estimate the motion of a rigid body in areas where no GPS information is available [13]. This method proposes to complement low-cost inertial sensors commonly used in navigation techniques (accelerometers and gyroscopes [5], [2]) with a specific device consisting of an array of spatially distributed magnetometers. The technique takes advantage of the unknown magnetic field disturbances usually observed indoor to reduce drift in velocities. This technique has been successfully applied for indoor laboratory experiments in [12]. Yet, down-scaling of the system (which is of paramount importance for embedded applications), and the need to substitute off-line computations and data treatments techniques with real-time computations has raised several important issues.

A first issue is the necessity of an accurate timing of the flow of data coming from the various subsystems of the distributed sensors set. An example of such distributed system is composed of nine 3 -axis sensors and produces a large amount of data under the form of 9 packets of 7 bytes every $6,5 \mathrm{~ms}$. The packets are sent according to the internal clocks of the sensors, which unavoidably are slightly inconsistent and result in missynchronization. In [4], a timestamping technique was proposed to address this issue.

A second issue results from the ill-calibration of the numerous sensors used. Consider, for example, a single 3axis magnetometer (e.g. a Honeywell $\AA$ HMR2300). As-is such an off-the-shelf sensor has several flaws. Its axis are

E. Dorveaux (corresponding author) is a $\mathrm{PhD}$ candidate in Mathematics and Control, Centre Automatique et Systèmes, Unité Mathématiques et systèmes, MINES ParisTech, 60, bd St Michel, 75272 Paris, France eric.dorveaux@mines-paristech.fr

D. Vissière is with SYSNAV, Zone industrielle B, 1 rue Jean de Becker Rémy, BP86, 27940 Aubevoye, France

N. Petit is with the Centre Automatique et Systèmes, Unité Mathématiques et systèmes, MINES ParisTech, 60, bd St Michel, 75272 Paris, France misaligned, and feature biases and scale factors. This results in measurement errors of the magnetic field. Fortunately, this problem can be dealt with by an appropriate calibration technique. Two classes of solutions exist. The first class, which is the most accurate but also the most tedious to implement, uses a so-called "calibration table" as reference system. A second class solely relies on the fact that, whatever the actual orientation of the sensor is, the norm of the sensed field should remain constant and equal to a reference value (e.g. the local Earth magnetic field which accurate database provide values of). By rotating the sensor in every possible direction, misalignments and biases can be identified. In [3] and [8], such methods were proposed. As all the "tablefree" calibration methods, this method has several practical advantages. In particular, it can be performed by the end-user of the navigation system and is often referred to as "on-thefield" method for this reason. This is of high interest since the low-cost sensors characteristics drift over time.

This calibration issue gets much more involved when several similar 3-axis sensors need to be used and their measurements need to be compared. This is the case in the navigation technique under consideration here. Consistency between these sensors is critical. This implies that the sensors must be calibrated and their calibrations must be consistent with each others. This is the topic under consideration in this paper.

The article is organized as follows. In Section I, the calibration problem is defined. Our focus is on magnetometers. The main defect of these systems (hard-iron, soft-iron) are briefly recalled and modeled with the classic biases, scale factors and misalignments. In Section II, three main strategies to calibrate a couple of sensors are presented. They can be easily generalized to any arbitrary number of sensors. The impact of a magnetic gradient, which is the main source of errors when putting the calibration procedure into practice, is studied for each strategy in Section III. Finally, in Section IV, the merits of the proposed calibration method are illustrated. An observer using the previously mentioned testbed to estimate the field and the velocity during translations is presented. Theoretical convergence of this observer is proved while simulation and experimental results are presented. Finally, we conclude and sketch future directions.

\section{CAlibration PROBLEM}

In this section, we recall the calibration problem for threeaxis sensors. We present an error model and the notations used throughout the paper. 
Consider a three-axis sensor. Denote $y_{p}(i)(3 \times 1$ vector) its sampled measurement, where $p$ stands for the sensor index and $i$ for the sampling index. This measurement is made when the sensor is at the (vector) position $x_{p}(i)$ where the actual sensed field is denoted $H\left(x_{p}(i)\right)$ shortened in $H_{p}(i)$ for sake of simplicity when there is no ambiguity.

The measurement errors can be modeled by constant coefficients of a (vector) affine transformation. The aim of the calibration process is to find the inverse affine transformation which maximizes a performance index. In this paper, the transformation is sought after under the form

$$
H_{p}(i)=A_{p} y_{p}(i)+B_{p}, \quad \forall i
$$

Here, $B_{p}$ is a zero-bias vector, and $A_{p}$ is referred to as the calibration matrix. $A_{p}$ accounts mainly for scale-factors, misalignments, and the resulting cross-coupling of axes. In the specific case of magnetometers, there are two other main errors to consider: hard and soft iron errors. $B_{p}$ accounts for hard iron errors, which are induced by permanent undesired fields (typically generated by ferromagnetic materials attached to the magnetometer frame) and result in a bias. As for soft iron errors, they are induced by materials that generate magnetic fields in response to externally applied magnetic fields. They generate an hysteresis phenomenon, which is often small enough to be neglected. $A_{p}$ takes into account the proportional part of this error. The constant of proportionality is referred to as the magnetic susceptibility of the considered material. The reader can refer to [10] for further details.

\section{A. Single sensor calibration}

Traditionally, calibration of three axis magnetometers is carried out in magnetically shielded facilities (see e.g. [10]). Measurements are performed in a precisely known magnetic field, and with precise knowledge of sensors orientation. However, the recent development of low-cost sensors has led to a paradox. Due to their relatively low quality, these lowcost sensors are in great need of calibration procedures, but the cost of the traditional procedures exceeds by many times the cost of the sensors themselves. Moreover, calibration parameters may change over time and on-the-field calibration is sometimes required. This has raised a huge interest in developing "simple but effective" calibration procedures that do not require a high degree of expertise nor an expensive hardware to be put into practice. Lately, some procedures and algorithms have been proposed for accelerometers (see [11], [6]) and magnetometers (see [8], [7], [3]) calibration. They all rely on the fact that the force field under consideration (respectively, the gravitational field and the Earth magnetic field) corresponds to a vector having, in theory, a constant and known norm. The calibration algorithm consists then, for sensor $p$, in finding the calibration matrix $A_{p}$ and bias $B_{p}$ such as the following index is minimized

$$
f\left(A_{p}, B_{p}, y_{p}\right)=\sum_{i=1}^{N}\left(\left\|A_{p} y_{p}(i)+B_{p}\right\|^{2}-1\right)^{2}
$$

This performance index involves the norm of the reconstructed data and a comparison against its theoretical (scalar) constant value (here 1 without loss of generality ${ }^{1}$ ). This function is quartic with respect to the coefficients of $A_{p}$ and $B_{p}$. In practice, the usual algorithm (see [8], [7]) proceeds in two steps. First, an exact linearization is performed by means of a change of variables. Then, an inverse transformation is analytically (or numerically) performed to obtain the desired variables. However, the linearizing change of coordinates is not unique. Several choices are possible and all yield some distortion in the cost function. The iterative algorithm proposed in [3] gets rid of this drawback by solving a sequence of least square problems in which the input data are iteratively calibrated. We now briefly recall this algorithm. Details on its properties (convergence and efficiency) can be found in [3].

Consider the $k^{t h}$ iteration. The $\mathrm{N}$ data under consideration are $y_{p, k}(i), i=1 . . N$ which are initialized at step $k=0$ with the measurements. The cost function to be minimized at this step is

$$
h\left(A, B, y_{p, k}\right)=\sum_{i=1}^{N}\left\|\left(A y_{p, k}(i)+B\right)-\frac{y_{p, k}(i)}{\left\|y_{p, k}(i)\right\|}\right\|^{2}
$$

This function is quadratic with respect to the coefficients of $A$ and $B$. A classic least-squares approach yields the uniquely defined solution

$$
\left(A_{p, k+1}, B_{p, k+1}\right)=\arg \min _{A, B} h\left(A, B, y_{p, k}\right)
$$

Data are then updated as follows using theses matrices

$$
y_{p, k+1}(i)=A_{p, k+1} y_{p, k}(i)+B_{p, k+1}
$$

After $k$ such iterations, a matrix $\tilde{A}_{p, k}$ and a bias vector $\tilde{B}_{p, k}$ are obtained, recursively, as

$$
\begin{aligned}
& \tilde{A}_{p, k}=A_{p, k} \tilde{A}_{p, k-1} \\
& \tilde{B}_{p, k}=A_{p, k} \tilde{B}_{p, k-1}+B_{p, k}
\end{aligned}
$$

They relate $y_{p, k}(i)$ to the raw measurements $y_{p}(i)$. In details,

$$
y_{p, k}(i)=\tilde{A}_{p, k} y_{p}(i)+\tilde{B}_{p, k}
$$

which represents the calibrated data of the $p^{t h}$ sensor at step $\mathrm{k}$.

\section{B. Obtaining experimental data}

Measurements are obtained while the sensor is oriented in every possible direction (see Figure 1 for a schematic planar illustration, the actual procedure involves three-dimensional rotations). No measurement of the sensor orientation is made during this data acquisition. Any calibration table is thus useless at this data collection step. The field under consideration (Earth Magnetic field for magnetometers, or gravitational field for accelerometers) is assumed to be constant during the data collection process. A warm-up phase (typically one

\footnotetext{
${ }^{1}$ This standpoint is different from numerous approaches found in the literature [1] where the norm of the sensed field is obtained from dependable look-up tables. No such information is available indoor.
} 


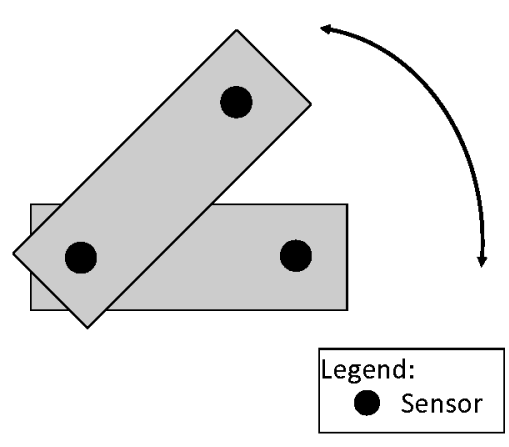

Fig. 1. In the calibration procedure, the sensor is rotated in every possible direction about its main axis.

or two minutes) has to be observed to avoid any temperature drift. The data acquisition phase itself lasts approximatively $25 \mathrm{~min}$ and a typical number of $N=10^{5}$ samples are considered.

\section{Theoretical CALibration of A SET OF TWO THREE-AXIS SENSORS}

In this section, we consider a set of two three-axis sensors (here magnetometers) to be calibrated, and expose three calibration methods. The methods discussed in this section can easily be extended to a wider array of sensors. The aim is to generalize the previously exposed simple but effective on-the-field calibration procedure used for a single sensor to an array of sensors.

The calibration of the sensor array consists in determining $A_{p}$ and $B_{p}$ for each sensor, such that

- each sensor considered separately is well calibrated, i.e. its norm is constant if it is rotated in a constant field,

- and all the sensors have the same output when they are put at the same location, i.e. their measurements should have the same norm and the same orientation.

An important issue is to get a data set where, for each sample, all the sensors measure the same field. This assumption may seem trivial, but in fact it implies two strong constraints on data acquisition: both sensors have to actually be exposed to the same field at the same date so that data from both sensors can be compared.

Firstly, as several sensors cannot be placed at the exact same location, data have to be acquired at a location where the sensed field is uniform. The impact of the presence of a gradient is discussed later on in Section III.

Secondly, both sensors have to make their measurements at the same dates. However, it is often not possible when using off-the-shelf digital sensors. There are two ways to get round this difficulty. The first one is to wait for a short period of time after each measurement point (i.e. to have quasi-static displacements). This allows to pick up at least one sample for each sensor at each measurement point. Such a procedure has a harmful impact on the duration of the data acquisition phase. The second way consists in interpolating the measurements at the same dates. This requires that sampling dates are precisely known. Such an acquisition technique is proposed in [4].

\section{A. Method 1: first sensor taken as reference}

This method proceeds in two steps. First, one of the two magnetometers is calibrated as a single sensor (following, e.g. the method recalled in $\S \mathrm{I}-\mathrm{A}$ ). Note $A_{1}$ and $B_{1}$ its calibration parameters. Then, the second magnetometer is calibrated using the newly calibrated measurements from the first sensor as references. The sought-after calibration parameters are noted $A_{2}$ and $B_{2}$. This second step is performed by minimizing the following cost function

$$
f_{1}\left(A_{2}, B_{2}\right)=\sum_{i=1}^{N}\left\|A_{2} y_{2}(i)+B_{2}-\left(A_{1} y_{1}(i)+B_{1}\right)\right\|^{2}
$$

This is a classical least-squares problem which, despite the large number $N$ of data, can be solved with very timeefficient algorithms. The first table-free calibration is run only once for the sensor taken as reference (resulting in the parameters $A_{1}$ and $B_{1}$ ). Then, a simple least-square algorithm is used for the second sensor. In the general case of an array of sensors, the least square-algorithm is run once for each sensor of the array.

B. Method 2: two independent calibrations followed by an harmonization step

Another method is as follows. First, both sensors are calibrated separately. Then, an harmonization step is performed so that both frames of coordinates have the same orientation. In other words, a rotation matrix relating the measurements from the calibrated sensors is calculated. In this last step, the function to minimize is detailed below in Equation (7). $O_{12}$ is the sought after rotation matrix, whereas $A_{1}, B_{1}, A_{2}$ and $B_{2}$ are the calibration parameters preliminarily obtained.

$$
f_{2}\left(O_{12}\right)=\sum_{i=1}^{N}\left\|O_{12}\left(A_{2} y_{2}(i)+B_{2}\right)-\left(A_{1} y_{1}(i)+B_{1}\right)\right\|^{2}
$$

\section{Method 3: Iterative method for simultaneous calibration}

A third method can be considered also. It extends the one proposed in [3] for a single 3-axes sensor. The iterative calibration is performed simultaneously for the two three-axis sensors. Consider a step in the iterations, say the $k^{\text {th }}$. We wish to account for the fact that the sensed field is constant and that it is the same for both sensors. Consider the $\mathrm{N}$ data $y_{p, k}(i), i=1, \ldots, N$ for the $p^{t h}$ magnetometer. They are initialized with the measurements at step $k=0$. First, we formulate the cost function to be minimized for sensor $p$, $p=1,2$.

$$
\begin{aligned}
& f_{p, 3}\left(A, B, y_{k}\right)= \\
& \sum_{i=1}^{N}\left\|\left(A y_{p, k}(i)+B_{p, k}(i)\right)-\frac{y_{1, k}(i)+y_{2, k}(i)}{2 \cdot\left\|y_{1, k}(i)+y_{2, k}(i)\right\|}\right\|^{2}
\end{aligned}
$$




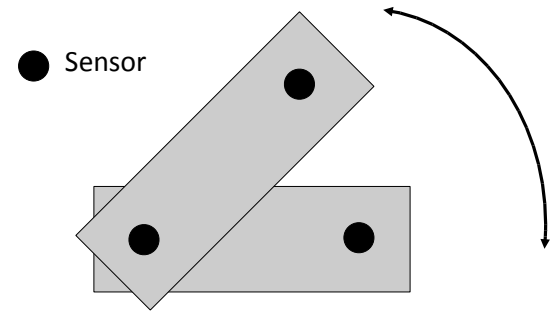

Fig. 2. When calibrating two sensors, rotation are made around one of the sensor, while the other one is moving on a sphere. The gradient of the sensed field has thus an impact on the measurements of the second sensor.

Importantly, this function is quadratic with respect to the coefficients of $A$ and $B$, yielding easy computation of the solution of the minimization of $f_{p, 3}$ with respect to these two arguments. For each sample, the value of the field taken as reference is the average $\left(\frac{y_{1, k}(i)+y_{2, k}(i)}{2 \cdot\left\|y_{1, k}(i)+y_{2, k}(i)\right\|}\right)$. It is the same for both sensors. We note the uniquely defined solution

$$
\left(A_{p, k+1}, B_{p, k+1}\right)=\operatorname{argmin}_{A, B} f_{p, 3}\left(A, B, y_{k}\right)
$$

which is obtained by a classic least-squares solver. Then, we use these matrices to update the data corresponding to each sensor $p$ as follows

$$
y_{p, k+1}(i)=A_{p, k+1} y_{p, k}(i)+B_{p, k+1}, \forall i=1, \ldots, N
$$

After $k$ such iterations, a matrix $\tilde{A}_{p, k}$ and a bias vector $\tilde{B}_{p, k}$ are obtained recursively for each sensor through

$$
\begin{aligned}
& \tilde{A}_{p, k}=A_{p, k} \tilde{A}_{p, k-1} \\
& \tilde{B}_{p, k}=A_{p, k} \tilde{B}_{p, k-1}+B_{p, k}
\end{aligned}
$$

They relate the calibrated data $y_{p, k}(i)$ to the raw measurements $y_{p}(i)$. Precisely,

$$
y_{p, k}(i)=\tilde{A}_{p, k} y_{p, k}(i)+\tilde{B}_{p, k}
$$

\section{IMPACT OF EXPERIMENTAL INACCURACIES}

The previous presented methods give good results in theory. Yet, several practical issues must be considered. In particular, in view of actual on-the-field magnetometers calibration which may be performed in slightly (or worse) magnetically perturbed areas, it is important to determine whether this disturbances will significantly impact on the calibration results. We now investigate the impact of a magnetic gradient at the place the calibration measurements are made. For sake of simplicity, we consider that two three-axis magnetometers are used. Except when explicitly mentioned, we assume, without any loss of generality, that the sensor three-dimensional rotations are made around the first magnetometer. This magnetometer is rotated strictly

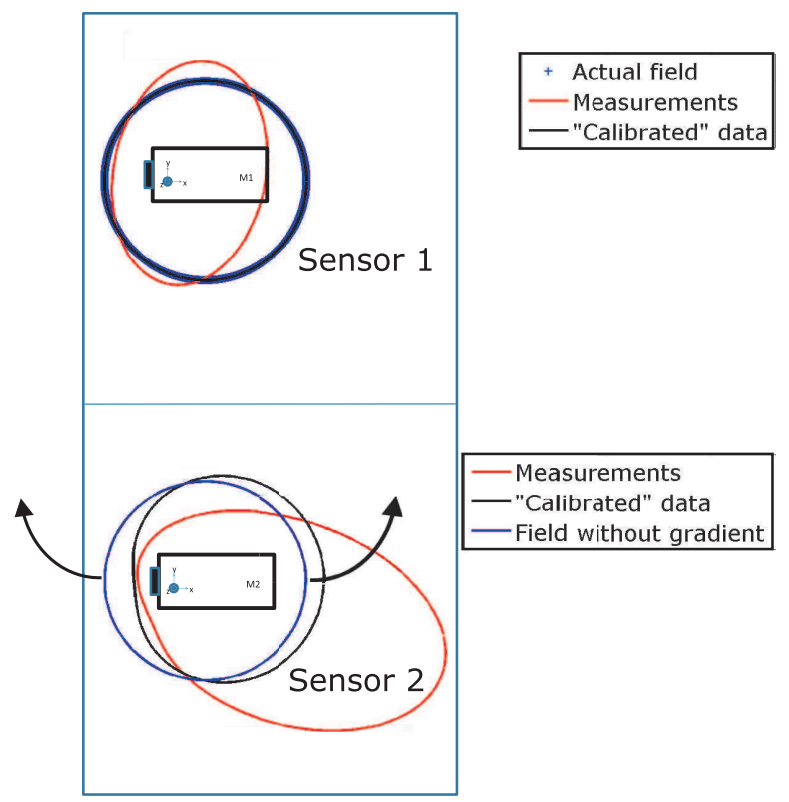

Fig. 3. Measurements and calibrated data for the two magnetometers Sensor 1 is placed at the center of rotation whereas sensor 2 is rotated around sensor 1 . Due to the nature of the center of rotation during the calibration, sensor 1 is well-calibrated while sensor 2 remains slightly illcalibrated.

around a fixed point. Therefore, it senses the exact same field during the rotations. By contrast, magnetometer 2, which is attached on the same board as magnetometer 1, is moving on a sphere centered on magnetometer 1 . For this reason, magnetometer 2 does not measure the exact same field during the calibration experiment because of the presence of the field gradient. This situation is pictured in Figure 2.

\section{A. Method 1}

As previously discussed, both sensors do not measure the exact same value of the field when its gradient is not zero. Consider $x_{1}(i)$ and $x_{2}(i)$ the (three dimensional) location of the sensors where the $i^{\text {th }}$ measurement is performed. According to the discussed calibration procedure, $x_{1}$ is constant while $x_{2}$ is moving on a sphere. Note the vector

$$
\delta_{12}(i)=x_{2}(i)-x_{1}(i)
$$

and the radius $\delta=\left\|\delta_{12}(i)\right\|$.

Note $H$ the (vector) sensed field which is not spatially constant. We have

$$
\begin{aligned}
H\left(x_{1}(i)\right) & =H\left(x_{2}(i)-\delta_{12}(i)\right) \\
& =H\left(x_{2}(i)\right)-\nabla H \delta_{12}(i)+o\left(\delta^{2}\right)
\end{aligned}
$$


The cost function to be minimized can be rewritten as follows

$$
\begin{aligned}
f_{1} & \left(A_{2}, B_{2}\right)=\sum_{i=1}^{N}\left\|A_{2} y_{2}(i)+B_{2}-H\left(x_{1}(i)\right)\right\|^{2} \\
& =\sum_{i=1}^{N}\left\|A_{2} y_{2}(i)+B_{2}-H\left(x_{2}(i)\right)+\nabla H \delta_{12}(i)\right\|^{2}+o\left(\delta^{2}\right) \\
& =\sum_{i=1}^{N}\left\|A_{2} y_{2}(i)+B_{2}-H\left(x_{2}(i)\right)+\epsilon_{12}(i)\right\|^{2}
\end{aligned}
$$

with

$$
\epsilon_{12}(i)=H \delta_{12}(i)+o(\delta)
$$

Let us note $\left(A_{2}^{*}, B_{2}^{*}\right)$ the parameters minimizing $f_{1}$ when $\delta$ is zero, i.e. the (ideal) calibration parameters when the field value is known at the precise location of the sensor. It is assumed to satisfy the following equation

$$
A_{2}^{*} y_{2}(i)+B_{2}^{*}=H\left(x_{2}(i)\right), \quad \forall i=1, \ldots, N
$$

A substitution in Equation (11) yields

$f_{1}\left(A_{2}, B_{2}\right)=\sum_{i=1}^{N}\left\|\left(A_{2}-A_{2}^{*}\right) y_{2}(i)+\left(B_{2}-B_{2}^{*}\right)-\epsilon_{12}(i)\right\|^{2}$

Consider

$$
E=\left(\begin{array}{cccc}
y_{2}(1)^{T} & 0 & 0 & \\
0 & y_{2}(1)^{T} & 0 & I_{3} \\
0 & 0 & y_{2}(1)^{T} & \\
\vdots & \vdots & \vdots & \vdots \\
y_{2}(N)^{T} & 0 & 0 & \\
0 & y_{2}(N)^{T} & 0 & I_{3} \\
0 & 0 & y_{2}(N)^{T} &
\end{array}\right)
$$

and, with $A^{\prime}=A-A^{*}$ and $B^{\prime}=B-B^{*}$, consider the vector of their components

$$
Z^{\prime}=\left(\begin{array}{lllllllll}
a_{1,1}^{\prime} & a_{1,2}^{\prime} & a_{1,3}^{\prime} & a_{2,1}^{\prime} & \ldots & a_{3,3}^{\prime} & b_{1}^{\prime} & b_{2}^{\prime} & b_{3}^{\prime}
\end{array}\right)^{T}
$$

Then, with

$$
\Sigma=\left(\epsilon_{12}(1)^{T} \epsilon_{12}(2)^{T} \ldots \epsilon_{12}(N)^{T}\right)^{T}+o(\delta)
$$

the cost function to be minimized can now be written

$$
f_{1}=\left\|E Z^{\prime}-\Sigma\right\|^{2}
$$

which is minimized by

$$
Z^{\prime}=\left(E^{T} E\right)^{-1} E^{T} \Sigma
$$

This last equation simply implies that the coefficients of the calibration parameters $\left(A_{2}, B_{2}\right)$ differ from the optimal ones $\left(A_{2}^{*}, B_{2}^{*}\right)$ (determined when the gradient of the sensed field is zero) by terms that are proportional to $\|\nabla H\|$.

\section{B. Method 2}

In the second method, both sensors are first calibrated separately before the harmonization step is performed. This requires two sets of data instead of a single one. The first set is obtained by rotating the system around magnetometer 1 and is used to calibrate this magnetometer, whereas the second set of data is acquired when rotating the whole system around magnetometer 2 in order to calibrate it. Of course, this procedure is more time-consuming, but it cancels the impact of the disturbances of the senses field onto the separate calibration of the sensors. Yet, the following harmonization step still suffers from the presence of a gradient, because the two distinct sets of data under consideration are slightly inconsistent. Further, we shall note that, in view of practical implementation, this method has two drawbacks. First, the time spent to acquire data for the calibration phase is proportional to the number of sensors in the array (which can be as large as 9 in the application considered in [4]). Including the necessary position shifts of the center of rotation of the whole system, this tedious work can take several hours. Secondly, it is difficult to perform the full rotations exactly around the center of each of the sensors, especially with custom built systems.

For these two reasons, we often prefer to leave out this second method in practical applications.

\section{Method 3}

In the third proposed method, the second magnetometer does not see a field of constant norm during the rotations. This error propagates through the formula

$$
\left(\frac{y_{1, k}(i)+y_{2, k}(i)}{2 \cdot\left\|y_{1, k}(i)+y_{2, k}(i)\right\|}\right)=H\left(x_{2}(i)\right)+\epsilon_{2, k}(i)
$$

where $\epsilon_{2, k}(i)$ vanishes when $x_{2}(i)=x_{1}(i)$. In this third method, both magnetometers are thus impacted by the sensed field disturbances in a way similar magnetometer 2 in to method 1 (but with a lower magnitude because of the averaging).

\section{Comparison of the proposed methods}

Method 1 is the quickest of the three proposed methods. It requires only one set of experimental data. However, the sensor taken as reference plays a special role. Changing this reference sensor leads to different calibration parameters. Method 2 is the longest one to put into practice: one set of data has to be acquired for each sensor. Moreover, it is difficult to actually make the rotations exactly around one sensor. But, if carefully performed, it is more accurate, especially regarding the bias. Finally, the third method requires only one set of data and lasts a bit longer than the previous one. However, all sensors play similar roles. The method is completely symmetric.

In summary, method 1 or 3 can be preferred, to minimize tedious calibration experiments, provided that the gradient of the sensed field remains reasonably small in the area where the sensors are to be calibrated. The effects of this gradient is also diminished if the sensors are located close 


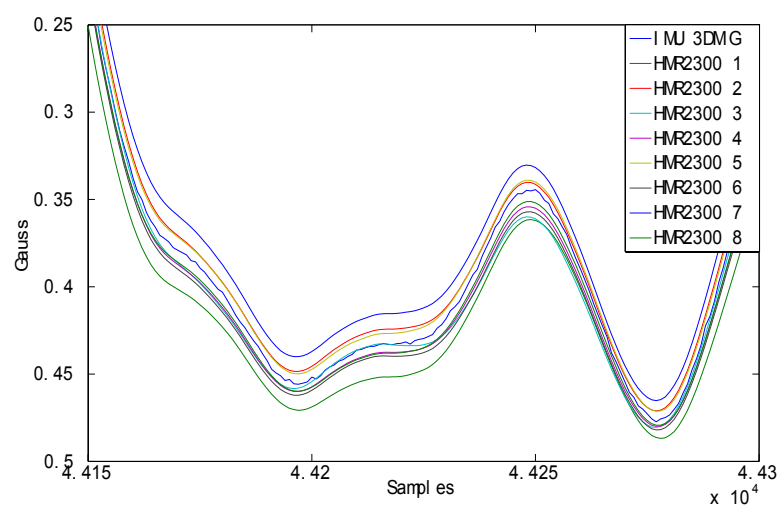

Fig. 4. The X-Components of 9 magnetometers calibrated separately in a place where the magnetic field is almost uniform. Inconsistencies can be observed.

to one another, though this is not always possible to set them up like this in practice. With these methods, an array of sensors can be efficiently and quickly calibrated using a single acquisition of data. A pictorial representation of the calibration results for method 1 is presented in Figure 3. In this simplified planar scheme, two sensors are considered and a full rotation is performed. Raw data which suffer from bias and misalignments take the form of two ellipses. The first sensor, which is the one around which the rotation is performed, can be completely calibrated. This yields a circle of calibrated measurements. On the other hand, due to presence of a non-zero gradient of the sensed field, the data of the second sensor can not be perfectly calibrated. This results is a close to circular set of calibrated data. The results presented here were obtained in simulation. The errors have been magnified to stress the role of the field disturbances.

These methods have also been applied on a vast set of magnetometers. We attached eight Honeywell@ HMR2300 magnetometers and one 3DMG-X1 from Microstrainß together and had the full system travel along an horizontal wooden rail. Prior to this, the magnetometers had all been calibrated separately. The data collected during this experiment are reported in Figure 4. They are inconsistent although the sensors were separately calibrated. This stresses the need of a joint calibration, as is proposed in all the methods of Section II. Then, the same experiment was conducted with sensors that were calibrated using method 3. The results obtained during this second experiment are reported in Figure 5. In this case, the results are consistent.

\section{EXAMPLE OF APPLICATION}

We wish to illustrate the potential of the sensing system that we developed by a simple case-study. Consider a set of two three-axis magnetometers that are attached together and move along a wooden rail. Once calibrated with one of the proposed calibration-table-free methods, they can be used to get an estimate of the local magnetic field and its gradient, by a finite difference scheme. These informations can be used in real time to estimate the translation velocity

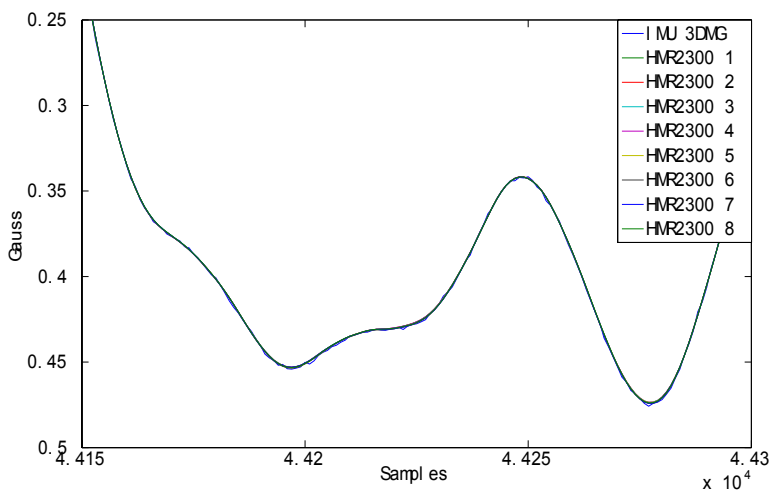

Fig. 5. The X-Component of 9 magnetometers calibrated all together in a place where the magnetic field is almost uniform. The data are consistent.

of a rigid body this system is attached to. Rotations can be accounted for with gyroscopes (see [13] for details).

For this purpose, we use an observer. The observer is first detailed in Section IV-A in a general case (translations in 3-D with an array of sensors allowing to completely estimate the Jacobian matrix of the magnetic field (at least 4 magnetometers are necessary)). A proof of convergence is given in Section IV-B. Finally, Experimental results are reported in Section IV-C. They stress the importance of the calibration step.

\section{A. Observer}

Again note $\mathrm{H}$ the magnetic field in a reference frame of coordinates, $J_{0}(H)$ its Jacobian, and $v_{0}$ the speed of the rigid body in this reference frame. If we consider only translations, we have

$$
\dot{H}=J_{0} v_{0}
$$

For the observer design, we assume that the velocity is constant, i.e.

$$
\dot{v}_{0}=0
$$

Measurements are made in the sensor frame of coordinates. Let $y$ be the measured magnetic field and $J$ the measured Jacobian (computed by a finite difference scheme from the discussed set of sensors). The sensor frame is not perfectly aligned with the reference frame. We note $R$ the constant rotation matrix from the reference frame to the sensor frame. With these notations, measurements equations are the following

$$
\begin{aligned}
& y=R H \\
& J=R J_{0}(H) R^{T}
\end{aligned}
$$

The dynamic model can then be rewritten in the sensor frame, $v=R v_{0}$ being the speed in that frame of coordinates

$$
\begin{aligned}
& \dot{y}=J v \\
& \dot{v}=0
\end{aligned}
$$


The following observer is built to estimate the field and the speed in the sensor frame.

$$
\begin{aligned}
& \dot{\hat{y}}=J\left[\hat{v}-L_{1}(\hat{y}-y)\right] \\
& \dot{\hat{v}}=-\ell_{2} J^{T}(\hat{y}-y)
\end{aligned}
$$

where $L_{1}$ is a matrix to be defined later on and $l_{2}>0$ is a constant parameter.

\section{B. Proof of convergence}

Consider the candidate Lyapunov-function $W$

$$
W=\|\hat{y}-y\|^{2}+\frac{1}{\ell_{2}} \cdot\|\hat{v}-v\|^{2}
$$

Its time-derivative is

$$
\dot{W}=-(\hat{y}-y)^{T}\left[J L_{1}+L_{1}^{T} J^{T}\right](\hat{y}-y)
$$

Choosing $L_{1}=J^{T}$ guarantees that $\dot{W}$ is negative semidefinite. If the set $\dot{W}(\hat{y}-y, \hat{v}-v)=0$ contains no other trajectory except the trivial one $(\hat{y}-y, \hat{v}-v)=(0,0)$, then, according to LaSalle's invariance principle [9], the origin is globally asymptotically stable. Let us verify that the invariant set is in fact reduced to the origin. Trajectories lying in the set $\dot{W}=0$ are such that

$$
J^{T}(\hat{y}-y)=0
$$

Assuming $\mathrm{J}$ has full rank, i.e. there are magnetic disturbances, we obtain

$$
\dot{\hat{y}}-\dot{y}=0
$$

However, $\dot{\hat{y}}-\dot{y}$ can be expressed as follows

$$
\dot{\hat{y}}-\dot{y}=J\left[\hat{v}-L_{1}(\hat{y}-y)\right]-J v
$$

which yields

$$
0=\hat{v}-v
$$

To conclude, if the Jacobian $J$ is a full-rank matrix, and in practice it usually is due to the field disturbances, the observer converges toward the value of the field and the velocity expressed in the frame of coordinates of the sensors.

\section{Experimental results}

We now implement the above presented observer in a simple one-dimensional case. Two magnetometers are used. From the velocity estimate given by the observer, an estimated position is then computed by a simple integration. The whole system is moved $1 \mathrm{~cm}$ forward every $4 \mathrm{~s}$.

Figure 6 shows experimental results when the system is moved forward for a few steps, then backward, step by step, until the original position is reached. The sensors are calibrated according to method 3 presented in this paper. Measurements are preliminarily filtered by a zero-phase digital filter and the synchronization technique discussed in [4] is applied. The observer is run on the resulting data. Figure 6(a) shows results when the two magnetometers are calibrated together. Both velocity and position estimates are accurate. In Figure 6(b), we report results obtained using the exact same technique but with separately calibrated magnetometers, i.e. ignoring the calibration methods proposed in this paper. The error is clearly visible on the gradient, which is sensitive since it is a differential measurement. This error leads to an increased speed and overestimated steps in position.
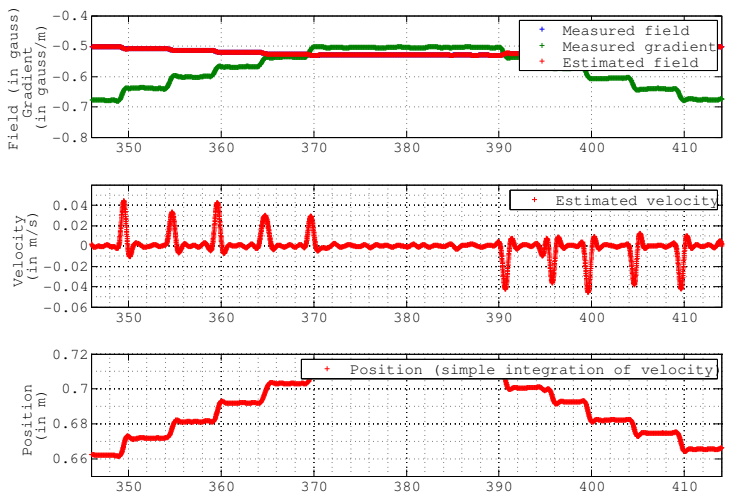

(a) Position and velocity estimates using sensors that have been calibrated together

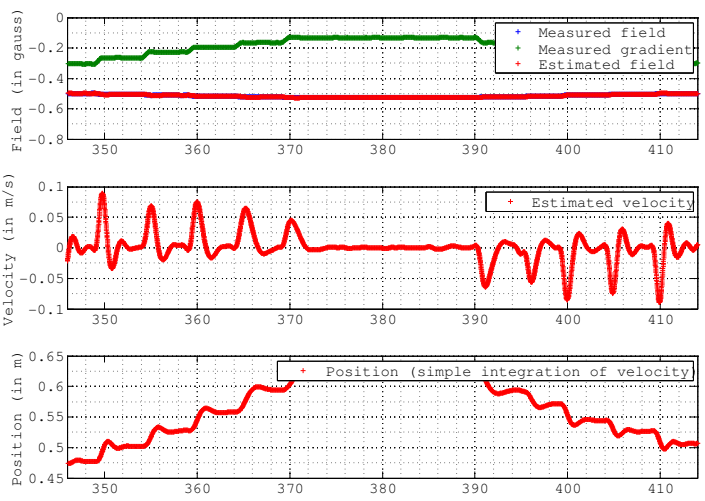

(b) Position and velocity estimates using sensors that have been calibrated separately

Fig. 6. Experimental results. The platform is translated step by step in direction sensors are lined up. One step of $1 \mathrm{~cm}$ is made every $5 \mathrm{~s}$, first forwards then backwards, after a rest of $20 \mathrm{~s}$.

\section{CONCLUSIONS AND FUTURE WORKS}

In this paper, we have proposed ways to extend the tablefree calibration method from one single sensor to a wider set of sensors. Three main strategies have been envisioned with some pros and cons. The main focus was on how to make these methods really effective in practice. This is why the impact of a gradient in the area where the data are acquired was studied. The theoretical efficiency of the methods can certainly be investigated further on. We also believe that additional calibration procedures may provide ways to complete the proposed method when the field is not perfectly uniform. These are current directions of future work. 


\section{ACKNOWLEDGMENTS}

This work was partially funded by the SYSTEM@TIC PARIS-REGION Cluster in the frame of project LOCINDOOR.

\section{REFERENCES}

[1] C. Barton. Revision of International Geomagnetic Reference Field Release. EOS Transactions, 77, April 1996.

[2] A. B. Chatfield. Fundamentals of High Accuracy Inertial Navigation, volume 174 of Progress in Astronautics and Aeronautics Series. AIAA, 1997.

[3] E. Dorveaux, D. Vissière, A. P. Martin, and N. Petit. Iterative calibration method for inertial and magnetic sensors. In Proc. of the 48th IEEE Conf. on Decision and Control, 2009.

[4] E. Dorveaux, D. Vissière, A. P. Martin, and N. Petit. Time-stamping for an array of low-cost sensors. In Proc. of the PDES 2009, IFAC workshop on Programmable Devices and Embedded Systems, 2009.

[5] P. Faurre. Navigation inertielle et filtrage stochastique. Méthodes mathématiques de l'informatique. Dunod, 1971.

[6] W. T. Fong, S. K. Ong, and A. Y. C. Nee. Methods for in-field user calibration of an inertial measurement unit without external equipment. Measurement Science and Technology, 19(8):085202-+, Aug. 2008.

[7] C. Foster and G. Elkaim. Extension of a non-linear, two-step calibration methodology to include non-orthogonal sensor axes. In IEEE Journal of Aerospace Electronic Systems, volume 44, July 2008.

[8] D. Gebre-Egziabher, G. Elkaim, J. Powell, and B. Parkinson. A nonlinear, two-step estimation algorithm for calibrating solid-state strapdown magnetometers. In 8th International St. Petersburg Conference on Navigation Systems (IEEE/AIAA), May 2001.

[9] H. K. Khalil. Nonlinear Systems. MacMillan, 1992.

[10] P. Ripka, editor. Magnetic Sensors and Magnetometers. ed. Boston: ARTECH HOUSE, 1. edition, 2001.

[11] I. Skog and P. Händel. Calibration of a mems inertial measurement unit. In XVII IMEKO World Congress Metrology for a Sustainable Development, Sept. 2006.

[12] D. Vissière, A. Martin, and N. Petit. Using spatially distributed magnetometers to increase IMU-based velocity estimation in perturbed areas. In Proc. of the 46th IEEE Conf. on Decision and Control, 2007.

[13] D. Vissière, A. P. Martin, and N. Petit. Using magnetic disturbances to improve IMU-based position estimation. In Proc. of the 9th European Control Conf., 2007. 